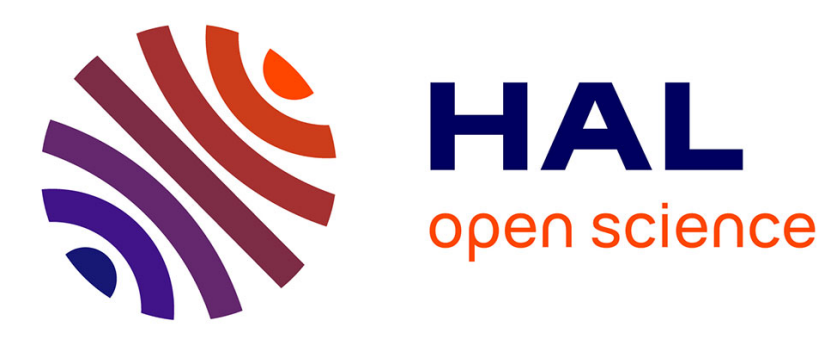

\title{
Matériaux pour dispositifs utilisant le déplacement de domaines magnétiques
}

\author{
J. Mareschal, D. Challeton, J. Daval, B. Ferrand, J.C. Gay
}

\section{To cite this version:}

J. Mareschal, D. Challeton, J. Daval, B. Ferrand, J.C. Gay. Matériaux pour dispositifs utilisant le déplacement de domaines magnétiques. Revue de Physique Appliquée, 1974, 9 (5), pp.865-868. 10.1051/rphysap:0197400905086500 . jpa-00243851

\section{HAL Id: jpa-00243851 https://hal.science/jpa-00243851}

Submitted on 1 Jan 1974

HAL is a multi-disciplinary open access archive for the deposit and dissemination of scientific research documents, whether they are published or not. The documents may come from teaching and research institutions in France or abroad, or from public or private research centers.
L'archive ouverte pluridisciplinaire HAL, est destinée au dépôt et à la diffusion de documents scientifiques de niveau recherche, publiés ou non, émanant des établissements d'enseignement et de recherche français ou étrangers, des laboratoires publics ou privés. 


\title{
MATÉRIAUX POUR DISPOSITIFS UTILISANT LE DÉPLACEMENT DE DOMAINES MAGNÉTIQUES
}

\author{
J. MARESChal, D. Challeton, J. DAVAl, B. FERRAND et J. C. GAY \\ L. E. T. I., C. E. N. G., B. P. no 85, 38041 Grenoble Cedex, France
}

\begin{abstract}
Résumé. - En nous appuyant sur des études menées au L. E. T. I. depuis plusieurs années, nous exposons les problèmes inhérents à l'obtention de matériaux pour dispositifs de mémorisation utilisant le déplacement de domaines magnétiques. Après un bref rappel du principe de fonctionnement de ces dispositifs et des performances à atteindre pour les rendre économiquement intéressants, nous décrivons les propriétés magnétiques statiques et dynamiques que doivent présenter les matériaux pour être utilisables. Facteur de qualité, longueur caractéristique, diamètre minimum et vitesse de déplacement des domaines, sont explicités en fonction des grandeurs magnétiques fondamentales.

Nous passons ensuite en revue les différents matériaux étudiés jusqu'ici et leur méthode de préparation. Les orthoferrites de terres rares, simples ou substitués, élaborés par différentes méthodes ont été les premiers monocristaux envisagés; ils ont été délaissés devant les difficultés rencontrées pour y abaisser au-dessous de quelques dizaines de microns le diamètre des domaines magnétiques. Des conclusions similaires ont été tirées d'études réalisées sur d'autres matériaux non cubiques. La fabrication, par épitaxie, de films d'oxydes mixtes à structure grenat dans lesquels une anisotropie uniaxiale est induite pendant la croissance, soit par l'établissement d'un gradient de concentration, soit par des contraintes magnétostrictives, permet de disposer de matériaux qui satisfont toutes les propriétés requises. Nous décrivons les trois méthodes de croissance épitaxique utilisées - solvants, transport en phase vapeur, synthèse hydrothermale - et en discutons les avantages et les difficultés à partir de résultats récents. Bien que les couches monocristallines obtenues actuellement soient pratiquement exemptes de défauts et aient atteint une qualité propre à l'industrialisation, ces matériaux restent d'élaboration délicate et coûteuse. Ces remarques justifient les recherches entreprises récemment pour remplacer les matériaux cristallisés étudiés jusqu'ici par des couches minces d'alliages ferrimagnétiques amorphes.
\end{abstract}

\begin{abstract}
Some problems about the search of materials for bubble domain memories are exposed from studies made at the L. E. T. I. since 1968. Operation principle and economical requirements of displays, static and dynamic magnetic properties of needed materials are briefly given. All materials and growth methods used up to now are reviewed. Single crystals of rare earth orthoferrites were among the first examined materials but the stable bubble size was too large. Similar difficulties were met with other non cubic materials. Heteroepitaxial garnet films with stress or growth induced uniaxial anisotropy have all the desired magnetic requirements. Problem of developing economic techniques for the growth of these epitaxial films is discussed from recent results on chemical vapor deposition, hydrothermal synthesis and liquid phase epitaxy. The single crystals films grown at present are of very good quality. But their high cost explains the present effort to replace them by thin amorphous ferrimagnetic films.
\end{abstract}

1. Introduction. - L'utilisation de domaines magnétiques cylindriques ou «bulles », présents dans certaines conditions dans des matériaux monocristallins uniaxes, pour réaliser de nouveaux dispositifs de logique et mémorisation a été imaginée en 1967 par Bobeck [1].

La théorie statique des domaines cylindriques a été proposée par Kooy et Enz [2] puis traitée plus en détail par Bobeck [1], Thiele [3], Callen et Josephs [4]. Elle montre que sous l'action d'un champ magnétique extérieur, des domaines cylindriques stables peuvent être créés dans une plaquette monocristalline possédant une forte anisotropie perpendiculairement à sa surface ; cette stabilité résulte de l'action de deux forces contraires, l'une générée par l'énergie magnétostatique, l'autre par l'énergie de paroi de domaine et par le champ appliqué.

Dans les utilisations qui nous intéressent, ces domaines sont générés, puis propagés parallèlement à la surface de la plaquette, sous l'influence d'un champ magnétique tournant agissant sur la polarité d'un dépôt magnétique à haute perméabilité de forme appropriée (Y - barre, $\mathrm{T}$ - barre, chevrons).

Pour chaque information binaire se présentant à l'entrée de la mémoire, le système génère ou ne génère pas un domaine, selon qu'il s'agit d'un « 1 » ou d'un « $0 »$; le domaine est ensuite propagé dans le circuit. La détection peut être accomplie en utilisant diffé- 
rents phénomènes : effet Hall, magnétorésistance, lecture optique.

Six ans après ses débuts, la technique des mémoires à « bulles » est parvenue à une relative maturité et l'on sait aujourd'hui comment fabriquer et organiser une mémoire de grande capacité [5-6] à partir de registres à décalages [7-8-9]. Nous nous contenterons d'évoquer, ici, l'aspect «matériau » de ces dispositifs : choix et élaboration.

2. Propriétés magnétiques requises pour les matériaux. - Elles sont fixées par les performances souhaitées pour les dispositifs. Il est communément admis que, pour être économiquement viables, les mémoires envisagées doivent pouvoir stocker une densité d'informations d'au moins $1,5 \times 10^{5} \mathrm{bits} / \mathrm{cm}^{2}$ avec un débit d'un million de bits par seconde.

Les travaux de Bobeck et Thiele ont permis de dégager un certain nombre de paramètres caractéristiques, reliables aux grandeurs magnétiques fondamentales, dont la considération est très utile pour guider le choix de l'élaborateur de matériaux. Ce sont en particulier la température de Curie, l'anisotropie, l'aimantation, le champ coercitif, la longueur caractéristique.

- LA Température de Curie, $T_{\mathrm{c}}$ détermine en grande partie les dérives thermiques des dispositifs. Des dérives faibles seront obtenues en utilisant des composés ferro- ou ferrimagnétiques présentant une température de Curie élevée, ou mieux, une température de compensation inférieure à la température ambiante.

- L'Aimantation à saturation, $M_{\mathrm{s}}$, fixe l'échelle des champs magnétiques de polarisation et de commande [10-3]. Elle doit être assez faible pour conserver des champs de valeur raisonnable, mais suffisamment grande pour que les problèmes de visualisation et de détection des bulles ne soient pas insolubles.

- Le CHAMP COERCITIF de paroi, $H_{c}$, impose l'espacement minimum entre domaines et par suite la densité de domaines utilisables. En fait, il doit être le plus faible possible, au moins inférieur à $1 \mathrm{Oe}$. Par ailleurs, $H_{\mathrm{c}}$ conditionne également la vitesse de déplacement d'un domaine donnée par la formule

$$
v=\frac{1}{2} \mu\left(\Delta H-\frac{8}{\Pi} H_{\mathrm{c}}\right)
$$

dans laquelle $\mu$ est la mobilité de paroi et $\Delta H$ le gradient de champ vu par le domaine.

- L'ANisotropie uniaxiale : l'existence de domaines magnétiques stables exige la présence d'une anisotropie uniaxiale, le champ d'anisotropie $H_{\mathrm{A}}$ devant satisfaire la relation

$$
H_{\mathrm{A}}>4 \pi M_{\mathrm{s}} .
$$

Le rapport $H_{\mathrm{A}} / 4 \pi M_{\mathrm{s}}$ est appelé le facteur de qualité du matériau. Cette anisotropie peut être structurale ou doit être induite dans les matériaux, en particulier lorsque ceux-ci sont isotropes (composés cubiques, amorphes). Deux solutions sont alors envisageables :

- Anisotropie de contrainte : elle résulte de la création d'une contrainte isotrope $\sigma$ dans le plan de la couche magnétique ; le champ d'anisotropie correspondant est donné par

$$
H_{\mathrm{A}}=\frac{\sigma \cdot \lambda}{M_{\mathrm{s}}}
$$

où $\lambda$ est une combinaison linéaire des coefficients de magnétostriction.

- Anisotropie de croissance : elle correspond à un abaissement de symétrie consécutif à la création d'un gradient de concentration parallèlement à une direction préférentielle de croissance.

- LA LONGUEUR CARACTÉRISTIQUe, $l$, résulte de considérations de stabilité magnétostatiques et relie les dimensions des domaines (diamètre, hauteur) aux propriétés magnétiques du matériau (constante d'échange $A$, énergie de paroi de domaine $\sigma_{\omega}$, anisotropie, aimantation) par la formule

$$
l=\frac{\sigma_{\omega}}{4 \pi M_{\mathrm{s}}^{2}}=\frac{(A K)^{1 / 2}}{\pi M_{\mathrm{s}}^{2}} .
$$

Ces considérations permettent de préciser les propriétés magnétiques que doivent présenter les matériaux pour être utilisables :

$$
\begin{aligned}
& T_{\mathrm{c}}>400 \mathrm{~K} \\
& 10^{2}<4 \pi M_{\mathrm{s}}>10^{3} \mathrm{G} \\
& 10^{2}>H_{\mathrm{A}}>10^{4} \text { Oe } \\
& 0,1 \times 10^{-6}<A< \\
& <1,6 \times 10^{-6} \mathrm{erg} / \mathrm{cm} \\
& \mu>200 \mathrm{~cm} / \mathrm{s} / \mathrm{Oe}
\end{aligned}
$$

Il y a lieu d'y rajouter la nécessité de tenir également compte de critères basés à la fois sur la facilité et sur le coût de l'élaboration.

3. Matériaux utilisés. - Un grand nombre de matériaux ont été évalués afin de les situer par rapport aux critères définis précédemment : orthoferrites des terres rares, ferrites hexagonaux, fluorure et borate de fer, ferrites spinelles et grenats, composés amorphes.

Bien qu'un travail considérable et la plupart du temps très astucieux ait été réalisé sur les premiers composés cités, en particulier sur les orthoferrites des terres rares à structure pérovskite déformée, les recherches correspondantes ont été abandonnées après avoir constaté qu'il n'était pas possible d'y ajuster tous les paramètres requis à des valeurs correctes : diamètres de domaines trop grands dans les orthoferrites [11-12], mobilité trop faible dans les ferrites hexagonaux [13], difficultés de fabrication trop importantes dans le fluorure et le borate de fer, aimantation trop forte dans les spinelles.

Une étape importante dans la recherche des maté- 
riaux utilisables a été franchie en 1970 avec la mise en évidence, dans des monocristaux de ferrites grenats élaborés dans un solvant, d'une anisotropie magnétique induite au cours de leur croissance [14]. L'ajustement des autres paramètres a été facilité par l'existence d'une abondante littérature traitant des propriétés physiques des grenats. Les formules complexes retenues par les différents laboratoires traduisent cette optimisation.

- LA SUBSTITUTION PARTIELLE, en sites tétraédriques, des ions fer par des ions gallium, permet d'ajuster la valeur de l'aimantation à quelques centaines de gauss tout en conservant une température de Curie suffisamment élevée ; selon la méthode de croissance utilisée, elle correspond en outre à des impératifs fixés par la fabrication.

- LA PRÉSENCE DE DEUX TERres RARES ou plus sur les sites dodécaédriques correspond à la nécessité de minimiser la dérive thermique des dispositifs au voisinage de la température ambiante en ajustant correctement la température de compensation. Leur choix et leur dosage judicieux permettent également d'agir sur l'aimantation, d'augmenter la mobilité et surtout, à partir des coefficients de magnétostriction, de contrôler l'anisotropie induite.

Compte tenu des dimensions souhaitées pour les domaines cylindriques $(\varnothing=6$ à $8 \mu)$, des considérations théoriques montrent que la couche magnétique doit avoir une épaisseur de quelques microns $(e \# \varnothing / 2)$. Dans ces conditions, une bonne tenue mécanique des échantillons ne peut pas être assurée par un amincissement des monocristaux massifs ; elle impose d'élaborer les systèmes retenus sous forme de films monocristallins hétéroépitaxiés sur un support diamagnétique approprié.

Quelle que soit la méthode d'épitaxie utilisée, le choix et la qualité du matériau support sont deux facteurs déterminants. Le premier fixe dans des limites étroites le paramètre cristallin du film à déposer et donc sa formule chimique. Le second conditionne la qualité finale de la couche épitaxiée puisqu'on a pu observer que tous les défauts présents dans le substrat étaient reproduits intégralement dans les films déposés. Le substrat le plus couramment utilisé est une plaquette monocristalline de gallate de gadolinium $\mathrm{Gd}_{3} \mathrm{Ga}_{5} \mathrm{O}_{12}$, obtenu par tirage à partir du produit fondu [15], dont la surface a subi une préparation très poussée. L'élaboration de ce matériau est compliquée par l'existence d'une évaporation non congruente [16] et par le décalage de la composition à fusion congruente par rapport à la composition $3: 5$ [17]. Un contrôle rigoureux de l'interface solide liquide pendant toute la croissance permet cependant d'obtenir des monocristaux pratiquement sans défauts. Seules y demeurent les stries de croissance résultant de l'allure du diagramme d'équilibre signalée ci-dessus ; ces défauts ne gênent pas le déplacement des domaines magnétiques.

Trois méthodes de croissance épitaxiques ont été utilisées pour élaborer les films de grenats : dépôt en phase vapeur, synthèse hydrothermale et surtout dépôt en phase liquide.

- LA MÉTHOde D'ÉPITAXIE PAR TRANSPORT EN PHASE VAPEUR [18] réalise la synthèse des composés désirés par oxydation des chlorures des constituants au voisinage du substrat maintenu à une température de $1200^{\circ} \mathrm{C}$. Elle a été presque partout abandonnée devant les difficultés rencontrées pour contrôler sur une surface suffisante l'épaisseur de la couche limite d'écoulement des gaz et pour limiter la croissance des germes d'oxydes dans la phase gazeuse ; ces inconvénients se traduisent par l'obtention de films d'épaisseur inégale et de composition variable.

- Plusieurs procédés ont été proposés [19-20] pour la croissance de films de grenat par synthèse hydrothermale. Les meilleurs résultats ont été obtenus en réalisant, vers $450^{\circ} \mathrm{C}$ et sous $1 \mathrm{kbar}$, à l'intérieur de l'autoclave, une réaction d'échange qui donne naissance au composé désiré et au solvant. Cela permet d'utiliser des concentrations initiales très faibles et de minimiser l'attaque du substrat avant la croissance [20]. Les films ainsi obtenus sont uniformes en composition et en épaisseur. La différence importante de solubilité entre le fer et le gallium dans les solvants basiques utilisés ne permet pas le dépôt de n'importe quelle composition et limite la technique à l'élaboration de compositions peu substituées (par exemple le système $(\mathrm{GdEr})_{3}(\mathrm{FeGa})_{5} \mathrm{O}_{12}$ avec seulement $\left.0,45 \mathrm{Ga}\right)$. Les résultats obtenus conduisent à l'obtention de films supportant des bulles inférieures à $4 \mu$. Compte tenu des remarques précédentes, l'intérêt de la synthèse hydrothermale est évident pour la fabrication de matériaux supportant des domaines de petits diamètres (mémoires à haute densité $=$ domaines de 1 à $2 \mu$ ). Cette méthode se prête de plus très bien à la fabrication de série, en utilisant de très grands autoclaves.

- L'ÉPITAXIE EN PHASE LIQUide dans des solvants à base d'oxyde de plomb reste la méthode la plus utilisée, parce que relativement facile à mettre en œuvre, au niveau de la recherche et du développement [21]. Comme dans les techniques précédentes, la stabilité de la phase grenat et la composition désirée sont fixées par les concentrations molaires initiales des différents constituants ; cette dernière est également très sensible aux paramètres de croissance que sont la température, la sursaturation et la vitesse de rotation du germe. D'autre part, alors que dans les deux autres méthodes l'anisotropie magnétique induite résulte exclusivement des contraintes provoquées par une accommodation élastique du film et du substrat, il s'y superpose éventuellement ici une anisotropie de croissance dont l'intensité varie avec les terres rares utilisées et en sens inverse de la température de dépôt. Une variation similaire de la quantité de plomb, en provenance du solvant, incorporée dans les sites dodécaédriques amène à proposer une relation de cause à effet entre la concentration de plomb et l'anisotropie de croissance. L'épitaxie en phase liquide permet d'élaborer tous les 
systèmes de grenats mixtes envisageables ; ceux retenus actuellement

$$
\left((\mathrm{SmY})_{3}(\mathrm{FeGa})_{5} \mathrm{O}_{12} \text { et }(\mathrm{EuY})_{3}(\mathrm{FeGa})_{5} \mathrm{O}_{12}\right)
$$

représentent des solutions intermédiaires dont l'anisotropie est induite par les deux mécanismes.

Bien que les couches monocristallines de grenats fabriquées au laboratoire par épitaxie en phase liquide aient atteint une qualité propre à l'industrialisation et que la méthode hydrothermale laisse apparaitre des possibilités de fabrication de grande série dans un futur assez proche, l'élaboration de ces matériaux reste délicate et coûteuse. Ces considérations économiques expliquent l'effort engagé récemment pour leur substituer des couches de matériaux amorphes dont on attend les avantages suivants :

- Techniques d'élaboration plus simples permettant de contrôler plus facilement les paramètres et les conduire ainsi à des coûts de fabrication plus faibles ;

- Elimination des problèmes liés aux défauts structuraux dont certains pourraient devenir gênants dans les applications futures mettant en œuvre des très petites bulles $(0,1 \mu)$;

- Possibilité de concevoir de véritables circuits intégrés mixtes électroniques magnétiques par dépôt de la couche amorphe sur une puce de silicium portant le circuit de commande.

L'existence du ferromagnétisme dans les composés amorphes a été prévue théoriquement et démontrée expérimentalement. La plupart des concepts définis dans l'état cristallisé restent valables et significatifs dans l'état amorphe. En particulier on a pu mettre en évidence des domaines magnétiques très analogues à ceux observés dans les cristaux.
En l'état actuel des connaissances, deux grandes familles de matériaux amorphes sont susceptibles d'être utilisés pour la réalisation de dispositifs à propagation de domaines magnétiques :

- L'une regroupe les alliages de terres rares et des métaux de transition de la famille du fer; les couches pouvant être préparées par pulvérisation cathodique sur un substrat refroidi [22].

- L'autre est constituée des alliages du type NiP ; leur propagation relève de méthodes chimiques ou électrochimiques simples à mettre en œuvre [23].

4. Conclusion. - La recherche et la fabrication des matériaux pour dispositifs utilisant le déplacement des domaines magnétiques cylindriques soulèvent un certain nombre de problèmes difficiles et quelques années de recherche et de développement sont encore nécessaires avant que les techniques soient définitivement au point.

La fabrication des grenats ferrimagnétiques par épitaxie en phase liquide est au point ; les problèmes résident dans la qualité du film et du substrat. Certains laboratoires savent aujourd'hui fabriquer des films sans défauts. Des résultats similaires devraient être obtenus par synthèse hydrothermale. L'intérêt de cette technique est étroitement lié aux progrès qui pourront être réalisés dans la technologie de dépôt des circuits de propagation. Dans l'hypothèse d'une miniaturisation de ces dépôts, les coûts de fabrication seront abaissés par les possibilités de fabrication de grandes séries qu'offre cette technique d'épitaxie.

$\mathrm{Au}$ niveau du matériau, la solution définitive pourrait cependant correspondre à l'utilisation des couches magnétiques amorphes en cours de mise au point dans différents laboratoires.

\section{Bibliographie}

[1] Boвeck, A. M., Bell. Syst. Tech. J. 46 (1967) 1901.

[2] Kooy, C., Enz, U., Philips Lab. 15 (1960) 7.

[3] Thiele, A. A., J. Appl. Phys. 41 (1970) 1139.

[4] Callen, H., Josephs, R. M., J. Appl. Phys. 42 (1971) 1977.

[5] Bonyard, P. et al., " Magnetic bubble memory chip design » (Intermag) 1973.

[6] Michaelis, P. et al., "Magnetic bubble mass memory》 (Intermag) 1973.

[7] Bonyard, P. et al., IEEE Trans. on Magnetics MAG-6 (1970) 447

[8] Bonyard, P., Nelson, T., Bell. Syst. Tech. J. 52 (1973) 307.

[9] Beausoleil, W. et al., IBM J. Res. Develop. (1972) 587.

[10] Thiele, A. A., Bell. System. Tech. J. 48 (1969) 3287.

[11] SHerwood, R. C. et al., Phys. Lett. 25A (1967) 297.
[12] Van Uitert, L. G. et al., Appl. Phys. Lett. 16 (1970) 84. [13] VAn Uitert, L. G. et al., Mat. Res. Bull. 5 (1970) 455.

[14] Kurzig, A. J., Hagedorn, F. B., IEEE Trans. Mag. MAG-7 (1971) 473.

[15] Brandle, C. A., Valentino, A. J., J. Cryst. Growth 12 (1972) 3.

[16] Carruthers, J. R, et al., J. Cryst. Growth 19 (1973) 204

[17] Allibert, M. et al., J. Cryst. Growth, à paraître.

[18] MeE, J. E. et al., IEEE Trans. Mag. MAG-5 (1969) 717.

[19] Kolb, E. D., Laudise, R. A., J. Appl. Phys. 42 (1971) 1552.

[20] Ferrand, B. et al., Mat. Res. Bull., à paraître.

[21] SHICK, L. H. et al., Appl. Phys. Lett. 18 (1971) 89.

[22] Electronic News, 12 février 1973.

[23] Proposition de marché DRME-LETI (1973). 